\title{
Identification of evolutionary relationships and DNA markers in the medicinally important genus Fritillaria based on chloroplast genomics
}

\author{
Tian Zhang ${ }^{1}$, Sipei Huang ${ }^{1}$, Simin Song ${ }^{1}$, Meng Zou ${ }^{1}$, Tiechui Yang ${ }^{2}$, Weiwei Wang ${ }^{1}$, Jiayu Zhou ${ }^{\text {Corresp., }}{ }^{1}$, Hai Liao \\ Corresp. 1 \\ ${ }^{1}$ School of Life Science and Engineering, Southwest Jiaotong University, Chengdu, Sichuan, China \\ 2 Qinghai Ivkang Biological Development Co., Ltd, Xining, Qinghai, China \\ Corresponding Authors: Jiayu Zhou, Hai Liao \\ Email address: spinezhou@home.swjtu.edu.cn, ddliaohai@home.swjtu.edu.cn
}

The genus Fritillaria has attracted great attention because of its medicinal and ornamental values. At least three reasons, including the accurate discrimination between various Fritillaria species, protection and sustainable development of rare Fritillaria resources as well as understanding of relationship of some perplexing species, have prompted phylogenetic analyses and development of molecular markers for Fritillaria species. Here we determined the complete chloroplast (CP) genomes for F. unibracteata, F. przewalskii, $F$. delavayi, and $F$. sinica through Illumina sequencing, followed by de novo assembly. The lengths of the genomes ranged from 151,076 in $F$. unibracteata to 152,043 in $F$. przewalskii. Those CP genomes displayed a typical quadripartite structure, all including a pair of inverted repeats $(26,078$ to $26,355 \mathrm{bp})$ separated by the large single-copy $(81,383$ to $81,804 \mathrm{bp})$ and small single-copy $(17,537$ to $17,569 \mathrm{bp})$ regions. Fritillaria przewalskii, $F$. delavayi, and $F$. sinica equivalently encoded 133 unique genes consisting of 38 transfer RNA genes, 8 ribosomal RNA genes, and 87 protein coding genes, whereas $F$. unibracteata contained 132 unique genes due to absence of the rps16 gene. Subsequently, comparative analysis of the complete CP genomes revealed that ycf1, trnL, trnF, ndhD, trnN-trnR, trnEtrnT, trnN, psbM-trnD, atpl, and rps19 to be useful molecular markers in taxonomic studies owning to their interspecies variations. Based on the comprehensive CP genome data collected from 53 species in Fritillaria and Lilium genera, a phylogenomic study was carried out with three Cardiocrinum species and five Amana species as outgroups. The results of the phylogenetic analysis showed that Fritillaria was a sister to Lilium, and the interspecies relationships within subgenus Fritillaria were well resolved. Furthermore, phylogenetic analysis based on the CP genome was proved to be a promising method in selecting potential novel medicinal resources to substitute current medicinal species that are on the verge of extinction. 
1 Identification of evolutionary relationships and DNA markers in the medicinally important genus

2 Fritillaria based on chloroplast genomics

3 Tian Zhang ${ }^{1}$, Sipei Huang ${ }^{1}$, Simin Song ${ }^{1}$, Meng Zou ${ }^{1}$, Tiechui Yang ${ }^{2}$, Weiwei Wang ${ }^{1}$, Jiayu Zhou ${ }^{1}{ }^{*}$, Hai Liao ${ }^{1}$

4

$5 \quad{ }^{1}$ School of Life Science and Engineering, Southwest Jiaotong University, Chengdu, Sichuan, China

$6 \quad{ }^{2}$ Qinghai lvkang Biological Development Co., Ltd., Xining, Qinghai, China

7 Authors' email address:

8 Tian Zhang: 1215695297@qq.com; Sipei Huang: 691786509@qq.com; Simin Song: 1158216140@qq.com;

9 Meng Zou: 815112542@qq.com; Tiechui Yang: 298237664@qq.com; Weiwei Wang: 1028694049@qq.com;

10 Jiayu Zhou: spinezhou@home.swjtu.edu.cn; Hai Liao: ddliaohai@home.swjtu.edu.cn.

$12 *$ For Correspondence

14 *Corresponding authors' information

15 Jiayu Zhou: spinezhou@home.swjtu.edu.cn;

16 Hai Liao: ddliaohai@home.swjtu.edu.cn 
19 Abstract: The genus Fritillaria has attracted great attention because of its medicinal and ornamental values.

20

21

At least three reasons, including the accurate discrimination between various Fritillaria species, protection and sustainable development of rare Fritillaria resources as well as understanding of relationship of some perplexing species, have prompted phylogenetic analyses and development of molecular markers for Fritillaria species. Here we determined the complete chloroplast (CP) genomes for $F$. unibracteata, F. przewalskii, F. delavayi, and F. sinica through Illumina sequencing, followed by de novo assembly. The lengths of the genomes ranged from 151,076 in $F$. unibracteata to 152,043 in F. przewalskii. Those CP genomes displayed a typical quadripartite structure, all including a pair of inverted repeats $(26,078$ to 26,355 bp) separated by the large single-copy (81,383 to 81,804 bp) and small single-copy (17,537 to 17,569 bp) regions. Fritillaria przewalskii, $F$. delavayi, and $F$. sinica equivalently encoded 133 unique genes consisting of 38 transfer RNA genes, 8 ribosomal RNA genes, and 87 protein coding genes, whereas $F$. unibracteata contained 132 unique genes due to absence of the rps16 gene. Subsequently, comparative analysis of the complete CP genomes revealed that $y c f 1, \operatorname{trn} L, \operatorname{trn} F, n d h D, \operatorname{trn} N-\operatorname{trn} R, \operatorname{trn} E-\operatorname{trn} T, \operatorname{trn} N, \operatorname{ps} b M-\operatorname{trn} D$, atpI, and rps 19 to be useful molecular markers in taxonomic studies owning to their interspecies variations. Based on the comprehensive CP genome data collected from 53 species in Fritillaria and Lilium genera, a phylogenomic study was carried out with three Cardiocrinum species and five Amana species as outgroups. The results of the phylogenetic analysis showed that Fritillaria was a sister to Lilium, and the interspecies relationships within subgenus Fritillaria were well resolved. Furthermore, phylogenetic analysis based on the CP genome was proved to be a promising method in selecting potential novel medicinal resources to substitute current medicinal species that are on the verge of extinction.

Abbreviations: CP, chloroplast; IR, inverted repeat; ITS, internal transcribed spacer; LSC, large single copy; SSC, small single copy; SSR, simple sequence repeats 


\section{Introduction}

The genus Fritillaria (Liliaceae), consisting of 140 known species, is widely distributed in Europe, Asia, and North America (Huang et al., 2018; Rix et al., 2001). Based on the Flora of China, twenty-two species are distributed throughout most provinces in China, among which four are diversity hotspots (Xinjiang Plain, East China Plain, Hengduan Mountains, and Northeast Plain). Fritillaria species have attracted much attention because they are widely used in traditional Chinese medicine and sometimes as ornamental plants. Dried bulbs from 11 species used singly or as components in traditional Chinese medicine preparations are recorded on Chinese Pharmacopeia (2020), and they are divided into five main concoctions, including Chuan-Bei-Mu (bulbs of the complex group of F. cirrhosa), Yi-Bei-Mu (bulbs of $F$. palilidiflora and $F$. walujewii), Zhe-Bei$\mathrm{Mu}$ (bulbs of $F$. thunbergii), Ping-Bei-Mu (bulbs of $F$. ussuriensis), and Hubei-Bei-Mu (bulbs of $F$. hupehensis). Although the bulb of each original species has its own unique curative effect and bioactive compounds and should be used separately for given purposes in traditional prescription, various Fritillaria species are still used indiscriminately in clinical prescription due to their similar morphology and names. Particularly, the morphological traits in the group that includes F. cirrhosa, F. unibracteata, F. przewalskii, F. delavayi, $F$. taipaiensis, and $F$. wabuensis, are extremely complex due to several highly variable characteristics including stem length, petal color, capsule winged or not, leaf curling, and scale number (Luo et al., 1996). Therefore, it is vital to carry out taxonomic identification of various Fritillaria species.

Molecular systematics has been widely used to clarify angiosperm phylogeny (Yang et al., 2016). Firstly, accurate identification (e.g., using DNA markers) has been necessary to discriminate between the Fritillaria species and its adulterants. Secondly, since the bulbs of some Fritillaria species show great economic values in Asian countries (Yeum et al., 2007) and have long been used in traditional Chinese medicine, the wild Fritillaria populations have experienced a sharp decline due to long-term overharvesting. To date, four species of Chuan-Bei-Mu and eight species in Xinjiang Plain have been classified as rare resources based on the list of rare endangered higher plants in China ( $L$ i et al., 2018). DNA markers have been helpful in understanding accurately the genetic diversity and structure of Fritillaria population, and thus can be an effective scientific approach for conservation purpose. Thirdly, a better understanding of the relationships within the genus could be of great significance for the medicinal use of Fritillaria. Some of the phylogenetically close species might 
be analyzed for their potential medicinal values to determine if they can be used as substitutes for species that are currently rare. Finally, the phylogenetic positions of some medicinal Fritillaria species, such as $F$. pallidiflora, F. wabuensis, and F. davidii, remain elusive. Fritillaria pallidiflora has always been considered a member of the subgenus Fritillaria by Rix (2001), whereas Rønsted et al (2005) linked it to the subgenus Petillium based on the results of molecular and morphological analyses. Fritillaria wabuensis was firstly discovered and nominated as a new species in Fritillaria (Tang \& Yue., 1983), but later it was classified as a variant of $F$. crassicaulis (Luo et al., 1996) and F. unibracteata (Liu et al., 2009), respectively. Therefore, well resolved molecular phylogenies of Fritillaria, especially the medicinal species, are necessary.

Currently, the genus Fritillaria is divided into eight subgenera, including Liliorhiza (including species mainly in North America), Japonica (including species mainly in Japan), Fritillaria (the biggest subgenus), Rhinopetalum, Petilium, the monotypic Davidii (including only F. davidii), Theresia (only F. persica), and Korolkowia (only F. sewerezowi), by Rix (2001). At present, despite the frequent usage of nuclear DNA internal transcribed spacer (ITS) and several plastid genome regions (trnL-trnF, matK, $r b c L$, and $r p l 16)$ in the classification of this genus, previous studies have found that these markers only provided weak phylogenetic signal. Rønsted et al (2005), who contributed to the current understanding of evolutionary relationships within Fritillaria, investigated the phylogenetic position of 37 Fritillaria species in detail using matK, rpll6 intron, and ITS. Consequently, Fritillaria was shown to be of two clades, with one clade mainly including species from the North American subgenus Liliorhiza and the other clade from the seven remaining subgenera. Consistent with the result of Rønsted et al (2005), Khourang et al (2014) revealed that the subgenus Fritillaria was a sister to the subgenus Rhinopetalum based on the phylogenetic tree constructed using the ITS and trnL$\operatorname{trnF}$ regions of nine Iranian species. However, Day et al (2014) showed that the largest subgenus (subgenus Fritillaria) appeared to be polyphyletic and formed two clades using $m a t K$ and $r b c L$ sequences, with one clade comprising taxa occurring mainly in Europe, the Middle East, Japan, and North Africa, and the other clade comprised taxa distributing in China and Central Asia. In our previous research, various Fritillaria species from China were classified as North China group and South China group based on the ITS2 sequences, but $57.1 \%$ of those species were not effectively resolved (Zheng et al., 2019). Recently, Li et al (2014) presented high-quality chloroplast genome using single molecule real-time sequencing, and suggested that rps 19 gene 
97

varied the greatest among various species. However, the noncoding regions showed higher variability and were potential effective molecular markers. Therefore, it was proposed that genomics based on the entire chloroplast genome sequences might help identify molecular markers with higher resolution (Xue et al., 2019).

The chloroplast (CP) genome has been extensively used for understanding phylogenetic relationships and discovering more effective molecular markers, some of which, such as $\operatorname{trnH}-\mathrm{ps} b A$, matK, and $r p l 16$, have been used as universal plant DNA barcodes (Bansala et al., 2018; Vinnersten \& Bremer., 2001). To date, there are 23 Fritillaria CP genomes that are available in GenBank and they can be used to enhance our understanding of the phylogenetic relationships and to identify molecular markers. Although previous reports (Bi et al., 2018; Chen et al., 2019; Chen et al., 2020; Huang et al., 2020; Park et al., 2017) performed comparative analyses with Fritillaria CP genomes available in GenBank, but species-specific identification has not been reported and the phylogenetic place of some ambiguous species remains elusive. At the initial stage of our study, the CP genomes of three important medicinal species ( $F$. unibracteata, F. przewalskii, and $F$. delavayi) and $F$. sinica had not been reported before 2018. The increasing CP genomes may not only provide a better phylogenetic analysis of this genus, but also promisingly promote the development of species-specific identification method in the future. Therefore, the $\mathrm{CP}$ genomes of these Fritillaria species were determined using the Illumina platform in the present study. The objectives of this study included (1) analyzing the global structural patterns of the four $\mathrm{CP}$ genomes and comparing them with the available $23 \mathrm{CP}$ genomes of Fritillaria; (2) assessing the phylogenetic relationships of the 11 medicinal species used in traditional Chinese medicine, so as to understand the phylogenetic position of some ambiguous species and find potential medicinal plants; and (3) obtaining candidate DNA markers (repeat sequences, SSRs, divergent regions, and indels).

\section{Materials and methods}

\section{Plant material}

The fresh leaves of $F$. unibracteata, F. przewalskii, $F$. delavayi and $F$. sinica were collected from the Huzhu County $\left(36^{\circ} 50^{\prime} 15^{\prime \prime} \mathrm{N}, 101^{\circ} 57^{\prime} 06^{\prime \prime} \mathrm{E}\right)$, Xining City, Qinghai Province, respectively. The Huzhu County is located in north Hengduan Mountains and east of the Qinghai-Tibetan Plateau. All samples were immediately frozen in liquid nitrogen and stored at $-80^{\circ} \mathrm{C}$ until DNA extraction. 


\section{Chloroplast genome sequencing and assembly}

Total genomic DNA was isolated from $100 \mathrm{mg}$ of fresh leaves using a modified CTAB method. The DNA concentration (>50 ng $\mu \mathrm{L}^{-1}$ ) was measured using a NanoDrop spectrophotometer. The isolated DNA was fragmented into small pieces using sonication. After end reparation and A-tailing, the short DNA fragments were ligated with the Illumina paired-end adaptors. Based on gel electrophoresis, the suitable fragments were purified and selected as templates for next-step PCR amplification to create the final DNA library. The quality and quantity of the DNA library were measured using the Agilent 2100 Bioanalyzer. Finally, the library was sequenced from both the 5' and 3' ends using Illumina NovaSeq6000 PE150 Sequencing platform (Illumina, CA, USA). By use of NGSQCToolkit v2.3.3, the raw reads were filtered to remove the linker sequence and low-quality reads defined as having more than $10 \%$ bases with Q-value $<20$, and thus high-quality clean reads were obtained. The clean reads were then assembled using SPAdes (Bankevich et al., 2012) 3.10 .1 (http://cab.spbu.ru/software/spades/) software with CP genome of $F$. cirrhosa as reference (NCBI accession number NC_024728.1). Finally, LSC/IR and SSC/IR junctions were further verified by Sanger sequencing.

\section{Genome annotation and sequence alignment}

In order to predict putative gene function, the CDS, rRNA and tRNA genes were aligned using blast v2.2.25 (https://blast.ncbi.nlm.nih.gov/Blast.cgi), HMMER v3.1b2 (http://www.hmmer.org/) and aragorn v1.2.38 (http://130.235.244.92/ARAGORN/), respectively, with $E$-value of $10^{-5}$. The OGDRAW (https://chlorobox.mpimp-golm.mpg.de/OGDraw.html) helped to make the $\mathrm{CP}$ genome maps of $F$. unibracteata, F. przewalskii, F. delavayi, and F. sinica.

The vmatch v2.3.0 (http: //www.vmatch.de/) could identify their scattered repetitive sequences (Askitis \& Sinha., 2010). MISA v1.0 (MIcroSAtellite identification tool, http://pgrc.ipk-gatersleben.de/misa/misa.html) helped to analyze CPSSR. The mafft v7.310 was used to perform indel identification (Katoh \& Standley., 2013). After using the mafft to align the $\mathrm{CP}$ genome sequences, BioEdit software was used to adjust the sequences manually (Gupta et al., 2014). DanSP v6.0 was used to perform sliding window analysis (step size $=200 \mathrm{bp}$ and window length=600 bp) for nucleotide variability (pi) in the whole CP genome (Rozas et al., 2017). 
152

153

154

155

156

157

158

159

160

161

162

163

164

165

166

167

168

169

170

171

172

173

174

175

176

177

\section{Phylogenetic analysis}

The phylogenetic analysis was firstly performed based on $m a t K$, psbA-trnH and $r p l 16$, respectively, by use of neighbor-joining (NJ) and maximum-likelihood (ML) methods. Then, the CP genomes in the phylogenetic analysis included the 27 Fritillaria species, 26 Lilium species, three Cardiocrinum species and five Amana species. The CP genome evolutionary tree was constructed by BLAST2OGMSA script (https://github.com/fenghen360/BLAST2OGMSA) (Bi., 2018) and MEGA-X software (Kumar et al., 2018). Firstly, multi-sequence alignment was conducted using BLAST tool of NCBI (Johnson et al., 2008), and then the initial alignment result was extracted by BLAST2OGMSA script to obtain homologous blocks. It was reported that BLAST2OGMSA relied on progressiveMauve, a kind of anchored alignment algorithm, to determine where locally collinear blocks (LCBs) represented the landmarks among organelle genomes (such as chloroplast and mitochondrial genomes). The co-exist LCBs among all organelle genomes were extracted and prepared for the further phylogenetic tree construction. In this study, the conserved CDS genes, functional noncoding regions, and rRNA genes as well were combined by BLAST2OGMSA. Finally, the alignment data from BLAST2OGMSA was imported into MEGA-X software to construct the phylogenetic tree using the NJ and ML methods, respectively.

\section{Results}

\section{Genome sequencing, assembly, and genome features}

Based on a stringent quality control, a total of 23,755,399 to 26,831,529 paired-end reads were obtained, generating 7,126,619,700 to $8,049,458,700$ clean bases data, from the four Fritillaria species. The resultant clean paired-end reads were then employed to assemble the $\mathrm{CP}$ genome using the complete genome sequence of $F$. cirrhosa as the reference. Totally, 471,385 to 652,632 mapping reads yielded an average coverage of 934X to $1292 \mathrm{X}$ for each species, generating four near full-length $\mathrm{CP}$ genomes that ranged from 151,076 in $F$. unibracteata to 152,043 in $F$. przewalskii. The CP genomes contained identical structures, such as two IR regions $(26,078$ to $26,355 \mathrm{bp}$ each) that were separated by a LSC region $(81,383$ to $81,804 \mathrm{bp})$ and a SSC region (17,537 to 17,569 bp) (Fig 1 and Table S1). 
A total of 133 genes were annotated, including 87 protein-coding genes (PCGs), 38 tRNA, and 8 rRNA genes. The global gene order and content were identical in the four species, except that $F$. unibracteata was absent of the rps 16 gene. 21 genes were duplicated in the CP genome, including eight tRNA genes, four rRNA genes, and nine PCGs. There were 13 genes containing introns, among which $c l p P$ and $y c f 3$ each had two introns, whereas the other 13 genes each had one intron. Eight, one, four, and two introns were located in the LSC, SSC, IRa, and IRb region, respectively (Table 1 and Table S2). Table S2 listed the 15 intron-containing genes in the CP genome of $F$. unibracteata, and those of $F$. przewalskii, $F$. delavayi, and $F$. sinica were included in Table S3, S4, and S5, respectively.

Four Fritillaria species showed high sequence similarity ( $>90 \%$ identity). IR regions showed a lower level of sequence divergence than LSC and SSC regions. Contraction and expansion of IR regions, especially the boundary region, are important aspects of $\mathrm{CP}$ genomes, which are the main reason of length variation in these genomes (Abdullah et al., 2020). As shown in Fig 2, these 12 Fritillaria species had the same gene contents and arrays in IR regions that were expanded in rps19 and ycfl genes. The rps19 gene in the 12 Fritillaria species crossed the LSC/IRb boundary and showed the same length of $279 \mathrm{bp}$ which was similar to that of Lilium superbum, except that F. cirrhosa had rps19 gene of $285 \mathrm{bp}$. In the LSC region, the length of rps 19 gene ranged from 250 to $268 \mathrm{bp}$, whereas that of rps 19 gene in the IRb region varied from 11 to $35 \mathrm{bp}$. Besides, the rps19 genes lost their protein-coding function due to incomplete gene duplication. The similar event was also observed in the $y c f 1$ gene at the IRb/SSC border. The $y c f l$ gene was largely located in the IRb and extended 16 to $32 \mathrm{bp}$ into the SSC region, whereas the $y c f 1$ gene in F. taipaiensis was fully located in the IRb region, $58 \mathrm{bp}$ from the IRb/SSC boundary. In the SSC/IRa boundary of 12 Fritillaria species, ycfl was a key gene and almost equally distributed. Ycfl gene had an SSC region of 4,320 bp in F. unibracteata and $F$. przewalskii, but 4,314 bp in F. delavayi, F. sinica, F. cirrhosa, and F. taipaiensis, and also had an IRa region of 1,230 bp in all species. By comparing the LSC/IRb, SSC/IRa, and IRa/LSC regions, it was found that there were obvious differences in IRb/SSC regions among the 12 Fritillaria species. The $y c f 1$ gene in $F$. taipaiensis and $F$. cirrhosa did not cross the IRb/SSC boundary, whereas those in the other Fritillaria species extended 16 to $32 \mathrm{bp}$ into the SSC region, which resulted a 16 to 32 bp overlap with $n d h F$ gene. 
Table 1

205

206

207

208

209

210

211

212

213

214

215

216

217

218

219

220

221

222

223

224

225

226

227

228

229

230

Fig. 1

Fig. 2

\section{Repeat sequence, simple sequence repeats (SSRs), divergent regions and indels}

The length of the repeat sequence ranged mainly from 15 to $20 \mathrm{bp}$ and rarely from 21 to $38 \mathrm{bp}$ among four Fritillaria species (Table S6). The repeating sequences were divided into forward repeating and palindrome sequences (including reverse and complementary sequences). The numbers of repeating sequences ranging from 15 to $20 \mathrm{bp}$ of $F$. unibracteata and $F$. przewalskii were more than 487, while those of $F$. delavayi and $F$. sinica were less than 350 (Fig S1). The numbers of repeat sequences in F. przewalskii, F. unibracteata, F. sinica, and $F$. delavayi were $1,200,976,656$, and 425 , respectively. Although repeat sequences with length ranging from 21 to $38 \mathrm{bp}$ were rare, several promising molecular markers were found. For instance, $F$. unibracteata had three forward repeats at lengths of 23,30, and $47 \mathrm{bp}$, respectively. Fritillaria delavayi also contained a palindromic repeat at a length of $54 \mathrm{bp}$, and $F$. przewalskii contained two forward repeats and a palindromic repeat at a length of $23 \mathrm{bp}$.

We also found $77,76,75$, and 72 SSRs of at least $10 \mathrm{bp}$ in F. przewalskii, F. sinica, F. unibracteata, and F. delavayi, respectively (Table S6, Fig 3). These SSRs were mainly located in the LSC region, followed by 50 SSRs in IR region, and a few SSRs in the SSC region. The single- and three-nucleotide SSRs were the majority detected in these Fritillaria species, the double-and four-nucleotide SSRs were the minority detected, and a few were five-nucleotide SSR. Single- and three-nucleotide repeats in F. unibracteata, F. przewalskii, F. delavayi, and $F$. sinica together accounted for $81.33 \%, 83.12 \%, 79.17 \%$, and $81.58 \%$ of SSRs, respectively. The single-nucleotide SSR with eight to nine repeated units were the most abundant and accounted for 53.91\% of SSRs (Fig 3). The high variation in numbers of SSRs might provide abundant information for molecular 
231

232

233

234

235

236

237

238

239

240

241

242

243

244

245

246

247

248

249

250

251

252

253

254

255

256

257

marker studies and plant breeding.

Fig. 3

Using slide window analysis, 16 regions were eventually extracted to calculate the nucleotide variability with pi value ranging from 0.0104 (rpl12) to 0.0159 (ycfl). The top ten most divergent regions were identified and thus might be used as potential molecular markers for future phylogenetic analysis and species identification in genus Fritillaria. These regions included $y c f 1, \operatorname{trn} L, \operatorname{trn} F, n d h D, \operatorname{trn} N$-trnR, trnE-trnT, trnN, psbM-trnD, atpI, and rps19 (Fig S2). Due to its highest divergence, ycf1 gene from 11 medicinal Fritillaria species was used to test its usefulness as a promising molecular marker for species identification. Based on our results, species-specific molecular markers for $F$. ussurinensis, $F$. pallidiflora, $F$. taipaiensis, $F$. walujewii, $F$. thunbergii, F. hupehensis, F. unibracteata, and F. delavayi were found (Fig S3). Fritillaria ussurinensis (78 SNPs and nine indels) had the highest number of molecular markers, whereas $F$. unibracteata (two SNPs), $F$. wabuensis (two SNPs), and F. delavayi (two SNPs and two indels) had the least number of molecular markers. However, species-specific markers for F. cirrhosa and F. przewalskii were not found in the $y c f l$ gene. For these two species, other highly divergent regions may provide better information for species-specific identification. Furthermore, due to its highest divergence, $y c f l$ gene was used to construct phylogenetic tree in the following section.

Also, three indels, including a 137-bp deletion within accD-psaI, a 47-bp insertion within trnG-GCCtrnR-UCU and a 6-bp deletion within intron of atpF, were found in F. taipaiensis, F. unibracteata, and $F$. cirrhosa $\mathrm{CP}$ genome, respectively. Inspringly, these indels provided a basis of species-specific identification despite the fact that further experiments should be needed in the future.

\section{Phylogenetic tree on the basis of $\mathrm{CP}$ genomes}

Prior to the phylogenetic analysis based on the CP genomes, we attempted to construct phylogenetic trees based on three common DNA barcodes from the CP genomes, including matK, psbA-trnH, and rpll6. Moreover, the $y c f 1$ gene was also used to construct the phylogenetic tree. As a result, matK, $p s b A$-trnH, and rpl16 obtained weakly supported trees, whereas phylogenetic tree based on $y c f 1$ gene was moderately 
258

259

260

261

262

263

264

265

266

267

268

269

270

271

272

273

274

275

276

277

278

279

280

281

282

283

284

supported as more than $50 \%$ and $60 \%$ branches got bootstrap values of more than 90 BP using NJ (Fig S4) and ML methods (Fig S5), respectively.

In addition, in comparison with four partial regions, the whole $\mathrm{CP}$ genomes obtained highly reliable phylogenetic tree. The CP genome matrix included the 27 Fritillaria species and 26 Lilium species, with three Cardiocrinum species and five Amana species as outgroups. On average, $(152,099)$ bp of the CP genome were aligned. The result of ML tree was similar to that of NJ tree (Fig S6). In the ML tree (Fig 4), the ingroup corresponding to Fritillaria and Lilium was strongly supported (100 BP), and were sisters to Cardiocrinum. In this analysis, Lilium was monophyletic (100 BP) and was a sister to Fritillaria. Furthermore, Lilium nested with Fritillaria with moderate bootstrap support (75 BP) than that (53 BP) of previous report (Day et al., 2014). Fritillaria, as the largest subgenus, was paraphyletic and majority of which fell into one strongly supported Eurasain clade (A) except F. maximowiczii (subgenus Liliorhiza). Within the clade A, F. davidii appeared as successive sister taxa to the remaining Eurasian species (100 BP), which split into two well-supported clades. Clade A1 grouped with the monotypic subgenus Rhinopetalum (F. karelinii) as a sister to two species from subgenus Fritillaria (F. ussuriensia and F. meleagroides), which occurred in North region of China. The sister clade (A2) was composed of the remaining 22 species that could be classified into two subclades (100 BP). Subclade B1 contained subgenus Theresia (F. persica) and subgenus Petilium (F. eduardii), which occurred in the Middle East and Central Asia, while subclade B2 comprising subgenus Fritillaria included 15 species from South China and five species (F. tortifolia, F. verticillata, $F$. yuminensis, $F$. pallidiflora, and $F$. walujewii) from Xinjiang Plain (Fig 5). The 11 most valuable species used in traditional Chinese medicine were not in the same monophyletic group, as $F$. ussuriensis was separated from the other 10 species. As a whole, the phylogenetic tree based on the CP genome was highly supported, in which 91\% (53 out of 58) branches obtained bootstrap values of more than $90 \mathrm{BP}$.

Fig. 4

Fig. 5 
285

286

287

288

289

290

291

292

293

294

295

296

297

298

299

300

301

302

303

304

305

306

307

308

309

310

311

\section{Discussion}

\section{The overall structure of the CP genome}

With the rapid development of de novo (Illumina) sequencing technology, the sequencing of CP genome is now cost-affordable and is much easier compared with the previous Sanger method. Moreover, de novo sequencing technology has been widely used in transcriptome assembly for identifying the biosynthetic and regulatory genes in traditional Chinese medicine, such as Ligusticum chuanxiong (Song et al., 2015) and Cassia obtusifolia (Deng et al., 2018). Here, four new CP genomes of Fritillaria were obtained using de novo sequencing technology. The CP genome sizes ranged from 151,076 to $152,043 \mathrm{bp}$, which were in accordance with those of reported CP genomes, such as F. ussuriensis (151,524 bp), F. taipaiensis (151,693 bp), and F.cirrhosa (151,991 bp). The four CP genomes contained similar genome structures, gene contents, and gene order, which were typical for land plants. Compared with the other three species, the number of $t R N A$ and $r R N A$ genes were the same, but the number of protein coding genes ranged from 77 to 78 due to the absence of the rps16 gene in F. unibracteata. The absence of rps16 gene has also been observed in Brassicaceae, Fabaceae, and Populus species (Jin et al., 2019). The functional loss of the rps16 gene from the CP genome could be compensated by the mitochondrial and (or) nuclear-encoded rps16 gene that could target chloroplast as well as mitochondria (Ueda et al., 2008).

The highly conserved genomic structure and gene order as well as no rearrangement of the Fritillaria CP genomes have been observed in previous reports. The $26 \mathrm{~kb}$ of IRs in the Fritillaria species was within the size range of most angiosperm CP genomes $(20$ to $30 \mathrm{~kb})$. The IR/LSC boundaries in the Fritillaria and Lilium (Lilium superbum) $\mathrm{CP}$ genomes expanded into the rps19 gene, which might be a characteristic CP genome structure of Fritillaria and its relative genus. Similar expansion was also observed in other taxa from family Liliaceae, including Lilium (Kim \& Lee., 2004), Fritillaria (Li et al., 2014), and Cardiocrinum (Liu et al., 2018). Li et al (2017) reported that the common location of IR/LSC junctions in the rps19 gene seemed to be an ancestral symplesiomorphy of Liliaceae. Here, similar feature was observed again, and the whole rps 19 gene was located inside the IR in Smilax china, Oncidium gower, and Allium chinense, while in Hordeum vulgare, the rps19 gene did not extend into the IR (Fig 2). The similar IRb/LSC boundaries among Fritillaria, Lilium, and Cardiocrinum implicated that these genera were closely related, which coincided with the 
312 phylogenetic result based on CP genomes (Fig 4).

313 A careful comparison between repeat sequence and SSR regions revealed significant differences between 314 various Fritillaria species, leading to the establishment of specific markers for molecular identification. In this 315 study, a large number of repeat sequences, mainly with lengths ranging from 15 to $20 \mathrm{bp}$, were detected in the 316 CP genomes of four Fritillaria species, consistent with the results on the CP genomes of Cannabaceae (Zhang 317 et al., 2018). SSRs have been used for the study of population genetics because of their high variability (Asaf 318 et al., 2016). The high ratio of SSRs in LSC region was also observed in F. sichuanica (Chen et al., 2019). In 319 the CP genomes of $F$. unibracteata, $F$. przewalskii, $F$. delavayi, and $F$. sinica, the contents of A/T repeats were much higher than those of G/C repeats, similar to the results of Xue et al (2019) and other studies (MelottoPassarin et al., 2011). Although several variable CP DNA markers, for instance, matK, $r p l 16$, atpB, and $r b c L$, have been used in phylogenetic studies of Fritillaria, but they showed small divergence (pi value of 0.00717 , $0.00571,0.00391$, and 0.00505 , respectively) among the 12 Fritillaria species. Based on the result of sliding window analysis, the top ten divergent regions were identified with pi value ranging from 0.0116 to 0.0159 . These highly divergent regions included $y c f 1, \operatorname{trn} L, \operatorname{trn} F, n d h D, \operatorname{trn} N$, atpI, and $r p s 19$ in the coding region, and $\operatorname{trnN}$-trnR, trnE-trnT, and $p s b M-t r n D$ in intergenic region. The molecular markers found in the $y c f 1$ gene indicated that the highly divergent regions provided plentiful information for species-specific identification in the future (Fig S3). Compared with the three common DNA barcodes, the $y c f 1$ gene generated a more reliable phylogenetic tree and it thus confirmed that highly divergent region was potential molecular markers for future phylogenetic analysis. The highly variable trnE-trnT and $y c f l$ gene have also been reported by Li et al (2018), and the $y c f l$ gene has been proposed as the most promising plastid DNA barcode of land plants (Dong et al., 2015).

\section{The phylogenetic analysis of medicinal genus Fritillaria}

Compared with partial sequences, the whole CP genome showed higher resolution with more than $91 \%$ et al., 2005) that increasing additional gene regions would help to improve the resolution. As suggested by 
339

340

341

342

343

344

345

346

347

348

349

350

351

352

353

354

355

356

357

358

359

360

361

362

363

364

365

resolve various Fritillaria species efficiently. Furthermore, our findings indicated that Fritillaria and Lilium were evidently sisters, with the closest relative being Cardiocrinum in a monophyletic genus (100 BP), similar with the result of Chen et al (2019). Such phylogenetic tree based on whole CP genome in this study showed similar topology with the previous study (Rønsted et al., 2005), but with higher resolution. Specially, genus Fritillaria was indicated as paraphyletic with higher bootstrap (100 BP) compared to 54 BP and 53 BP in the findings of Rønsted et al (2005) and Day et al (2014), respectively.

The subgenus Fritillaria also appeared to be a paraphyletic group, similar to the results of Day et al (2014). One important medicinal Fritillaria species, F. ussuriensis, clustered with F. meleagroides and formed a sister clade to F. karelinii of subgenus Rhinopetalum, similar to the results of Huang et al (2020), Khourang et al (2014), and Li et al (2018). Fritillaria ussuriensis and F. meleagroides were frequently considered as members of the large subgenus Fritillaria (Rix 2001). However, the two species do have some similarities with F. karalinii as both of them have small mastoid on filament, which are different from other species in Xinjiang Plain with no mastoid. Similar conflict between molecules and morphology was also observed in other taxa (Anand et al., 2016). Meanwhile, such mastoid on filament was proposed to be a potential primitive feature, and our results partly supported this hypothesis because $F$. karelinii and F. meleagroides diverged early from other medicinal species from Xinjiang Plain, such as F. pallidiflora and $F$. walujewii. Subgenus Theresia $(F$. persica) and Petilium (F. eduardii) had close relationship and formed monophyletic subclade B1, which was similar to the results of Day et al (2014) and Li et al (2018).

As shown in Fig 4 and Fig 5, five species from Xinjiang Plain were included in a strongly supported subclade C1 (Fig 4), which was a sister to subclade C2 containing the other 15 species from outside Xinjiang Plain. This signified that the Xinjiang species had a close genetic relationship. All the four species that were distributed in East China Plain, including F. monantha, F. anhuiensis, F. thunbergii, and F. hupehensis, nested in a supported subclade (100 BP). The remaining 11 species, including the complex group of $F$. cirrhosa, in another subclade were distributed in Hengduan Mountains (100 BP). The 11 important medicinal Fritillaria species were widely distributed in four hotspots, namely Xinjiang Plain, Northeastern China Plain, East China Plain, and Hengduan Mountains. The former two regions constituted hotspots in North China, while the latter two regions constituted hotspots in South China. Interestingly, the eight species in the upper location of the 
366

367

368

369

370

371

372

373

374

375

376

377

378

379

380

381

382

383

384

clade originated from Xinjiang Plain and Northeastern China Plain (F. ussuriensis), whereas the 12 species in the lower location distributed in East China Plain and Hengduan Mountains region. Consequently, the geographical distribution pattern of the 11 medicinal species appeared to map on the phylogenetic tree, especially by plastid data (Rønsted et al., 2005). Similar result was also reported by Li et al (2018), and thus the investigation on the correlation between distribution pattern and phylogenetic relationship was needed in the future.

Early in 1987, F. unibracteata, F. cirrhosa, F. prezewalskii, and $F$. delavayi were recorded as national third-class endangered medicinal plants in China (Konchar et al., 2011). The most important medicinal species showed close relationship to widely cultivated members of subgenus Fritillaria, which raised the possibility of the rare species being replaced by those widely cultivated species. Recent analyses have demonstrated that $F$. crassicaulis, showing closest relationship with $F$. cirrhosa, has been widely used as the substitution of $F$. cirrhosa by people of Naxi nationality and Tibetan since Ming/Qing Dynasty (Tang \& Xue., 1992). These findings highlighted those phylogenetic trees based on the CP genomes were promising in selecting potential novel medicinal species. In the future, those showing close relationship to the important species in traditional Chinese medicine, such as $F$. sichuanica, $F$. dajinensis, $F$. yuzhongensis, $F$. sinica, and $F$. crassicaulis, should be investigated to determine if these bulbs contain the same bioactive compounds found in the complex group of F. cirrhosa.

\section{The phylogenetic placement of some Fritillaria species}

The non-monophyletic trait of subgenus Fritillaria indicated the incongruence in classification among some species, similar to the reports by Rønsted et al (2005) and Day et al (2014). Although F. ussurinensis was regarded as a member of subgenus Fritillaria, its splitting from other members of subgenus Fritillaria has also been observed by Chen et al (2019) and Huang et al (2020). There were several reports of natural interspecific hybrids (e.g., F. ussurinensis (Ruan et al., 2004) and F. eduardii (Wietsma et al., 2011)), which might promote the molecular phylogenetic non-monophyly (Funk \& Omland., 2003). Secondly, F. davidii had rice-shaped bulbils, resembling the morphological character of subgenus Liliorhiza, and used to be grouped in subgenus Liliorhiza. But based on our results, it was distantly related to subgenus Liliorhiza and was thus 
393

394

395

396

397

placed in subgenus Davidii as described by Rix (2001). It was suggested that rice-shaped bulbils have independently evolved in $F$. davidii and subgenus Liliorhiza due to geographic separation, followed by a loss in some species in Eurasian clade during evolution (Rønsted et al., 2005). Thirdly, Rønsted et al (2005) found that F. pallidiflora was resolved solely within the Korolkowia/Petilium/Theresia clade by combining plasmid rpl16 and matK sequences. Our results demonstrated that $F$. pallidiflora clustered within subgenus Fritillaria and was more closely related to Petilium/Theresia. The conflict in F. pallidiflora was likely to be solved by using whole $\mathrm{CP}$ genome instead of separate regions. In addition, based on the $\mathrm{CP}$ genome, $F$. unibraceata was a sister to $F$. wabuensis with a divergence of 0.003 , which was more than that between $F$. sichuanica and $F$. dajinensis (0.002). If $F$. sichuanica and $F$. dajinensis were given at rank of species, it was preferable to follow Tang \& Yue (1983) and to rank F. wabuiensis as species instead of rank of variant. However, this result was merely based on the $\mathrm{CP}$ genome, the accurate placement of $F$. wabuensis will be kept for further evaluation by nuclear genome comparison although it is extremely difficult to obtain.

\section{Conclusion}

The CP genomes of the four Fritillaria species were useful resources for taxonomic clarification, determination of phylogenetic relationship and development of DNA markers. The phylogenetic tree based on the whole $\mathrm{CP}$ genome was reliable since $91 \%$ branches obtained bootstrap values of more than $90 \mathrm{BP}$, and the result supported the monophyly of genus Lilium, Amana and Cardiocrinum, except that the largest genus Fritillaria was paraphyletic. The 11 members of subgenus Fritillaria that were used in traditional Chinese medicine were split into two clusters since $F$. ussuriensis clustered with $F$. meleagroides and $F$. karelinii. In addition, the phylogenetic tree appeared to reflect a geographic distribution pattern of subgenus Fritillaria, and also highlighted the importance of the $\mathrm{CP}$ genome in the evolutionary analysis. The most important medicinal species, especially the complex group of $F$. cirrhosa, were found to be close to species that were in widespread cultivation for medicinal and ornamental purposes. Excitingly, those closely related species from subgenus Fritillaria might be promising alternatives to balance the improving market and rare resources. Finally, this study provided comprehensive molecular markers that might be valuable for future establishment of speciesspecific identification in Fritillaria. 
Availability of data and materials

The chloroplast genomes generated during the current study were deposited in NCBI with accession number of MW849272 (F. unibraceata), MW849274 (F. przewalskii), MW849275 (F. delavayi) and MW849273 $(F$.

sinica), respectively. All the raw Illumina data of F. unibracteata, F. przewalskii, F. delavayi and F. sinica have been deposited in the Sequence Read Archive (SRA) of the NCBI under accession numbers of SRR14454932, SRR14455034, SRR14454929 and SRR14455331, respectively.

\section{References}

Abdullah, Henriquez CL, Mehmood F, Carlsen MM, Islam M, Waheed MT, Poczai P, Croat TB, Ahmed I. 2020. Complete chloroplast genomes of Anthurium huixtlense and Pothos scandens (Pothoideae, Araceae): unique inverted repeat expansion and contraction affect rate of evolution. $J$ Mol Evol 88(7): 562-574. DOI: 10.1007/s00239-020-09958-w

Anand KK, Jena SN, Chaudhary LB, Singh M. 2016. Conflict between morphological and molecular data: a case study of Ficus krishnae (Moraceae). Phytotaxa 247: 143-147. DOI: https://doi.org/10.11646/phytotaxa.247.2.7.

Angen Ø, Johannesen TB, Petersen RF, Uldum SA, Schnee C. 2021. Development of a species-specific real-time PCR test for Chlamydia psittaci and its employment in the investigation of zoonotic transmission from racing pigeons in Denmark. Diagn Microbiol Infect Dis 100(2): 115341. DOI: 10.1016/j.diagmicrobio.2021.115341.

Asaf S, Khan AL, Khan AR, Waqas M, Kang SM, Khan MA, Lee SM, Lee IJ. 2016. Complete chloroplast genome of Nicotiana otophora and its comparison with related species. Front Plant Sci 7: 843. DOI: $10.3389 /$ fpls.2016.00843.

Askitis N, Sinha R. 2010. RepMaestro: scalable repeat detection on disk-based genome sequences. Bioinformatics 26(19): 2368-74. DOI: 10.1093/bioinformatics/btq433.

Bankevich A, Nurk S, Antipov D, Gurevich AA, Dvorkin M, Kulikov AS, Lesin VM, Nikolenko SI, Pham S, Prjibelski AD, Pyshkin AV, Sirotkin AV, Vyahhi N, Tesler G, Alekseyev MA, Pevzner PA. 
2012. SPAdes: a new genome assembly algorithm and its applications to single-cell sequencing. $J$ Comput Biol 19(5): 455-77. DOI: 10.1089/cmb.2012.0021.

Bansal S, Thakur S, Mangal M, Mangal AK, Gupta RK. 2018. DNA barcoding for specific and sensitive detection of Cuminum cyminum adulteration in Bunium. Phytomedicine 50(15): 178-183. DOI: 10.1016/j.phymed.2018.04.023.

Bi GQ. 2018. BLAST2OGMSA. Available at https://github.com/fenghen360/BLAST2OGMSA.

Bi Y, Zhang MF, Xue J, Dong R, Du YP, Zhang XH. 2018. Chloroplast genomic resources for phylogeny and DNA barcoding: a case study on Fritillaria. Sci Rep 8: 1184. DOI: 10.1038/s41598-018-19591-9.

Chen Q, Wu XB, Zhang DQ. 2019. Phylogenetic analysis of Fritillaria cirrhosa D. Don and its closely related species based on complete chloroplast genomes. Peer J 7: e7480. DOI: 10.7717/peerj.7480.

Chen Q, Wu XB, Zhang DQ. 2020. Comparison of the abilities of universal, super, and specific DNA barcodes to discriminate among the original species of Fritillariae cirrhosae bulbus and its adulterants. PLoS One 15(2): e0229181. DOI: 10.1371/journal.pone.0229181.

Day PD, Berger M, Hill L, Fay MF, Leitch AR, Leitch IJ, Kelly LJ. 2014. Evolutionary relationships in the medicinally important genus Fritillaria L. (Liliaceae). Mol Phylogenet Evol 80: 11-19. $\quad$ DOI: 10.1016/j.ympev.2014.07.024.

Deng Y, Zheng H, Yan Z, Liao D, Li C, Zhou J, Liao H. 2018. Full-length transcriptome survey and expression analysis of Cassia obtusifolia to discover putative genes related to aurantio-obtusin biosynthesis, seed formation and development, and stress response. Int $J$ Mol Sci 19: 2476. DOI: 10.3390/ijms 19092476 .

Dong W, Xu C, Li C, Sun J, Zuo Y, Shi S, Cheng T, Guo J, Zhou S. 2015. Ycf1, the most promising plastid DNA barcode of land plants. Sci Rep 5: 8348. DOI: 10.1038/srep08348.

Funk DJ, Omland KE. 2003. Species-level paraphyly and polyphyly: frequency, causes and consequences with insights from animal mitochondrial DNA. Ann Rev Ecol Evol Syst 34: 397-423.

Gupta SK, Padmanabhan BR, Diene SM, Lopez-Rojas R, Kempf M, Landraud L, Rolain JM. 2014. ARG-ANNOT, a new bioinformatic tool to discover antibiotic resistance genes in bacterial genomes. Antimicrob Agents Chemother 58(1): 212-20. DOI: 10.1128/AAC.01310-13. 
474

475

476

477

478

479

480

481

482

483

484

485

486

487

Huang J, Yang LQ, Yu Y, Liu YM, Xie DF, Li J, He XJ, Zhou SD. 2018. Molecular phylogenetics and historical biogeography of the tribe Lilieae (Liliaceae): Bi-directional dispersal between biodiversity hotspots in Eurasia. Ann Bot 122: 1245-1262. DOI: 10.1093/aob/mcy138.

Huang J, Yu Y, Liu YM, Xie DF, He XJ, Zhou SD. 2020. Comparative chloroplast genomics of Fritillaria (Liliaceae), inferences for phylogenetic relationships between Fritillaria and Lilium and plastome evolution. Plants (Basel) 9(2): 133. DOI: 10.3390/plants9020133.

Jin DP, Choi IS, Choi BH. 2019. Plastid genome evolution in tribe Desmodieae (Fabaceae: Papilionoideae). PLoS One 14(6): e0218743. DOI: 10.1371/journal.pone.0218743.

Johnson M, Zaretskaya I, Raytselis Y, Merezhuk Y, McGinnis S, Madden TL. 2008. NCBI BLAST: a better web interface. Nucleic Acids Res 36: W5-9. DOI: 10.1093/nar/gkn201.

Katoh K, Standley DM. 2013. MAFFT multiple sequence alignment software version 7: improvements in performance and usability. Mol Biol Evol 30(4): 772-80. DOI: 10.1093/molbev/mst010.

Khourang M, Babaei A, Sefidkon F, Naghavi MR, Asgari D, Potter D. 2014. Phylogenetic relationship in Fritillaria spp. of Iran inferred from ribosomal ITS and chloroplast trnL-trnF sequence data. Biochem Syst Ecol 57: 451-457. DOI: 10.1016/j.bse.2014.10.001.

Kim KJ, Lee HL. 2004. Complete chloroplast genome sequences from Korean ginseng (Panax schinseng Nees) and comparative analysis of sequence evolution among 17 vascular plants. DNA Res 11: 247-261. DOI: $10.1093 /$ dnares/11.4.247.

Konchar K, Li XL, Yang YP, Emshwiller E. 2011. Phytochemical variation in Fritillaria cirrhosa D. Don (Chuan Bei Mu) in relation to plant reproductive stage and timing of harvest. Econ Bot 65: 283-294. DOI: 10.1007/s12231-011-9170-3.

Kress WJ, Wurdack KJ, Zimmer EA, Weigt LA, Janzen DH. 2005. Use of DNA barcodes to identify flowering plants. P Natl Acad Sci USA 102: 8369-8374. DOI: 10.1073/pnas.0503123102.

Kumar S, Stecher G, Li M, Knyaz C, Tamura K. 2018. MEGA X: molecular evolutionary genetics analysis across computing platforms. Mol Biol Evol 35(6): 1547-1549. DOI: 10.1093/molbev/msy096.

Li P, Lu RS, Xu WQ, Ohi-Toma T, Cai MQ, Qiu YX, Cameron KM, Fu CX. 2017. Comparative genomics and phylogenomics of east Asian Tulips (Amana, Liliaceae). Front Plant Sci 8: 451-463. DOI: 
10.3389/fpls.2017.00451.

502

503

504

505

506

507

Li Q, Li Y, Song J, Xu H, Xu J, Zhu Y, Li X, Gao H, Dong L, Qian J, Sun C, Chen S. 2014. Highaccuracy de novo assembly and SNP detection of chloroplast genomes using a SMRT circular consensus sequencing strategy. New Phytol 204(4): 1041-9. DOI: 10.1111/nph.12966.

Li Y, Zhang Z, Yang J, Lv G. 2018. Complete chloroplast genome of seven Fritillaria species, variable DNA markers identification and phylogenetic relationships within the genus. PLoS One 13(3): e0194613. DOI: 10.1371/journal.pone.0194613.

Liu HY, Yu Y, Deng YQ, Li J, Huang ZX, Zhou SD. 2018. The chloroplast genome of Lilium henrici: genome structure and comparative analysis. Molecules 23(6): 1276. DOI: 10.3390/molecules23061276.

Liu ZD, Wang S, Chen SC. 2009. A taxonomic note of Fritillaria wabuensis (Liliaceae). Acta Botanica Yunnanica (in Chinese) 31(2): 145.

Luo YB, Chen XQ. 1996. A revision of Fritillaria L. (Liliaceae) in the Hengduan Mountains and adjacent regions, China (II). Acta Phytotaxonomica Sinica (in Chinese) 34(5): 547-553.

Melotto-Passarin DM, Tambarussi EV, Dressano K, De Martin VF, Carrer H. 2011. Characterization of chloroplast DNA microsatellites from Saccharum spp and related species. Genet Mol Res 10: 2024-2033. DOI: $10.4238 /$ vol10-3gmr1019.

Ng PK, Lin SM, Lim PE, Liu LC, Chen CM, Pai TW. 2017. Complete chloroplast genome of Gracilaria firma (Gracilariaceae, Rhodophyta), with discussion on the use of chloroplast phylogenomics in the subclass Rhodymeniophycidae. BMC Genomics 18(1): 40. DOI: 10.1186/s12864-016-3453-0.

Park I, Kim WJ, Yeo SM, Choi G, Kang YM, Piao R, Moon BC. 2017. The complete chloroplast genome sequences of Fritillaria ussuriensis Maxim. and Fritillaria cirrhosa D. Don, and comparative analysis with other Fritillaria species. Molecules, 22(6): 982. DOI: 10.3390/molecules22060982.

Rix EM. 2001. Fritillaria: A revised classification together with an updated list of species. Publication of the Fritillaria Group of the Alpine Garden Society UK.

Rønsted N, Law S, Thornton H, Fay MF, Chase MW. 2005. Molecular phylogenetic evidence for the monophyly of Fritillaria and Lilium (Liliaceae; Liliales) and the infrageneric classification of Fritillaria. Mol Phylogenet Evol 35(3): 509-27. DOI: 10.1016/j.ympev.2004.12.023. 
528

529

530

531

532

533

534

535

536

537

538

539

540

541

542

543

544

545

546

547

548

549

550

551

552

553

554

Rozas J, Ferrer-Mata A, Sánchez-DelBarrio JC, Guirao-Rico S, Librado P, Ramos-Onsins SE, SánchezGracia A. 2017. DnaSP 6: DNA sequence polymorphism analysis of large data sets. Mol Biol Evol 34(12): 3299-3302. DOI: 10.1093/molbev/msx248.

Ruan HL, Zhang YH, Pan XC, Dong T, Wu JZ. 2004. Studies on the chemical constituents from culbs of hybridized Bulbus Fritillariae ussuriensis. Zhongguo Zhong Yao Za Zhi (in Chinese) 29(4): 331-334.

Song T, Liu ZB, Li JJ, Zhu QK, Tan R, Chen JS, Zhou YU, Liao H. 2015. Comparative transcriptome of rhizome and leaf in Ligusticum Chuanxiong. Plant Syst Evol 301: 2073-2085.

Tang SY, Yue SJ. 1983. Three new species of Fritillaria Linn. Acta Academiae Medicinae Sichuan (in Chinese) 14(4): 327-334.

Tang SY, Yue SJ. 1992. Fritillaria genus, Flora of Sichuan. Publication of Sichuan Ethnic Publishing House 7: 55-82.

Ueda M, Nishikawa T, Fujimoto M, Takanashi H, Arimura S, Tsutsumi N, Kadowaki K. 2008. Substitution of the gene for chloroplast RPS16 was assisted by generation of a dual targeting signal. Mol Biol Evol 25(8): 1566-75. DOI: 10.1093/molbev/msn102.

Vinnersten A, Bremer K. 2001. Age and biogeography of major clades in Liliales. Am J Bot 88(9): 1695-703.

Wietsma WA, van den Berg RG, van Scheepen J, Wieringa JJ 2011. The nomenclatural history of Fritillaria eduardii and the correct names of its varieties. TAXON 60(6): 1754-1759. DOI: 10.1002/tax.606018.

Xue S, Shi T, Luo W, Ni X, Iqbal S, Ni Z, Huang X, Yao D, Shen Z, Gao Z. 2019. Comparative analysis of the complete chloroplast genome among Prunus mume, P. armeniaca, and P. salicina. Hortic ResEngland 6: 89. DOI: 10.1038/s41438-019-0171-1.

Yang Y, Zhou T, Duan D, Yang J, Feng L, Zhao G. 2016. Comparative analysis of the complete chloroplast genomes of five Quercus species. Front Plant Sci 7: 959. DOI: 10.3389/fpls.2016.00959.

Yeum HS, Lee YC, Kim SH, Roh SS, Lee JC, Seo YB. 2007. Fritillaria cirrhosa, Anemarrhena asphodeloides, Lee-Mo-Tang and cyclosporine a inhibit ovalbumin-induced eosinophil accumulation and Th2-mediated bronchial hyperresponsiveness in a murine model of asthma. Basic Clin Pharmacol Toxicol 100(3): 205-13. DOI: $10.1111 /$ j.1742-7843.2007.00043. x. 
555

556

557

558

559

560

561 $(1$

Zhang HL, Jin JJ, Moore MJ, Yi TS, Li DZ. 2018. Plastome characteristics of Cannabaceae. Plant Divers 40(3): 127-137. DOI: 10.1016/j.pld.2018.04.003.

Zheng H, Deng KY, Chen AQ, Fu SB, Zhou D, Wang WW, Ni DM, Ren YY, Zhou JY, Liao H. 2019. Molecular identification and genetic relationship of Fritillaria cirrhosa and related species based on DNA barcode. Acta Pharmaceutica Sinica (in Chinese) 54(12): 2326-2334.

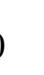


Figure 1

Chloroplast genome maps of F. unibracteata, F. przewalskii, F. delavayi and F. sinica.

Fig 1. Chloroplast genome maps of $F$. unibracteata, F. przewalskii, F. delavayi and F. sinica. Genes belonging to functional group are color-coded. The positive coding gene is located on the outside of the circle, and the reverse coding gene is located on the inside of the circle. The grey circle inside circle represents the GC content.

photosystem I photosystem II cytochrome b/f complex $\square$ ATP synthase NADH dehydrogenase RubisCO large subunit RNA polymerase ribosomal proteins (SSU) ribosomal proteins (LSU) clpP, matK other genes hypothetical chloroplast reading frames (ycf) ORFs

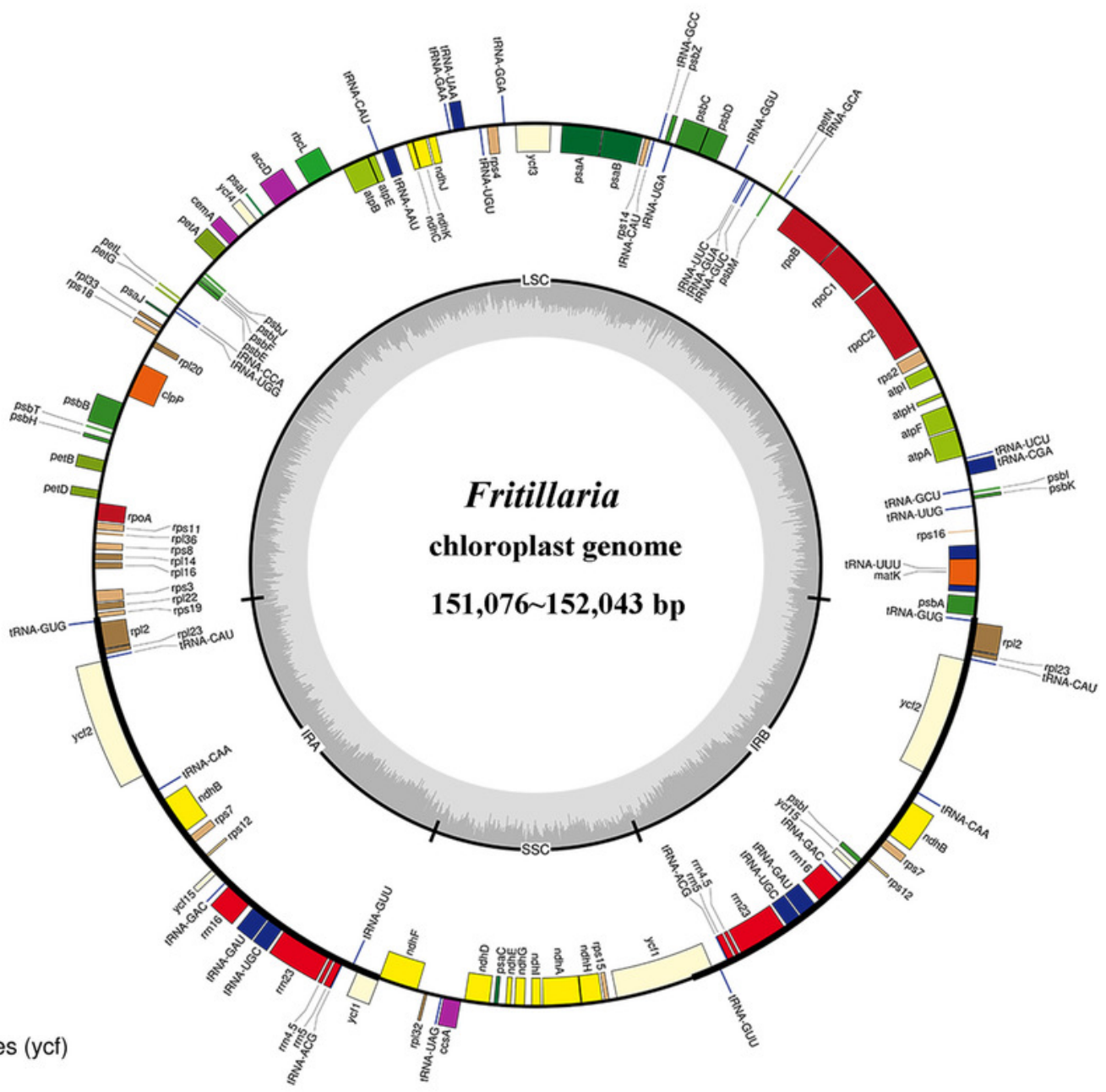

transfer RNAs

ribosomal RNAs

origin of replication 
Figure 2

Comparison of LSC, IRs, and SSC junction positions among 17 CP genomes.

Fig 2. Comparison of LSC, IRs, and SSC junction positions among 17 CP genomes. 


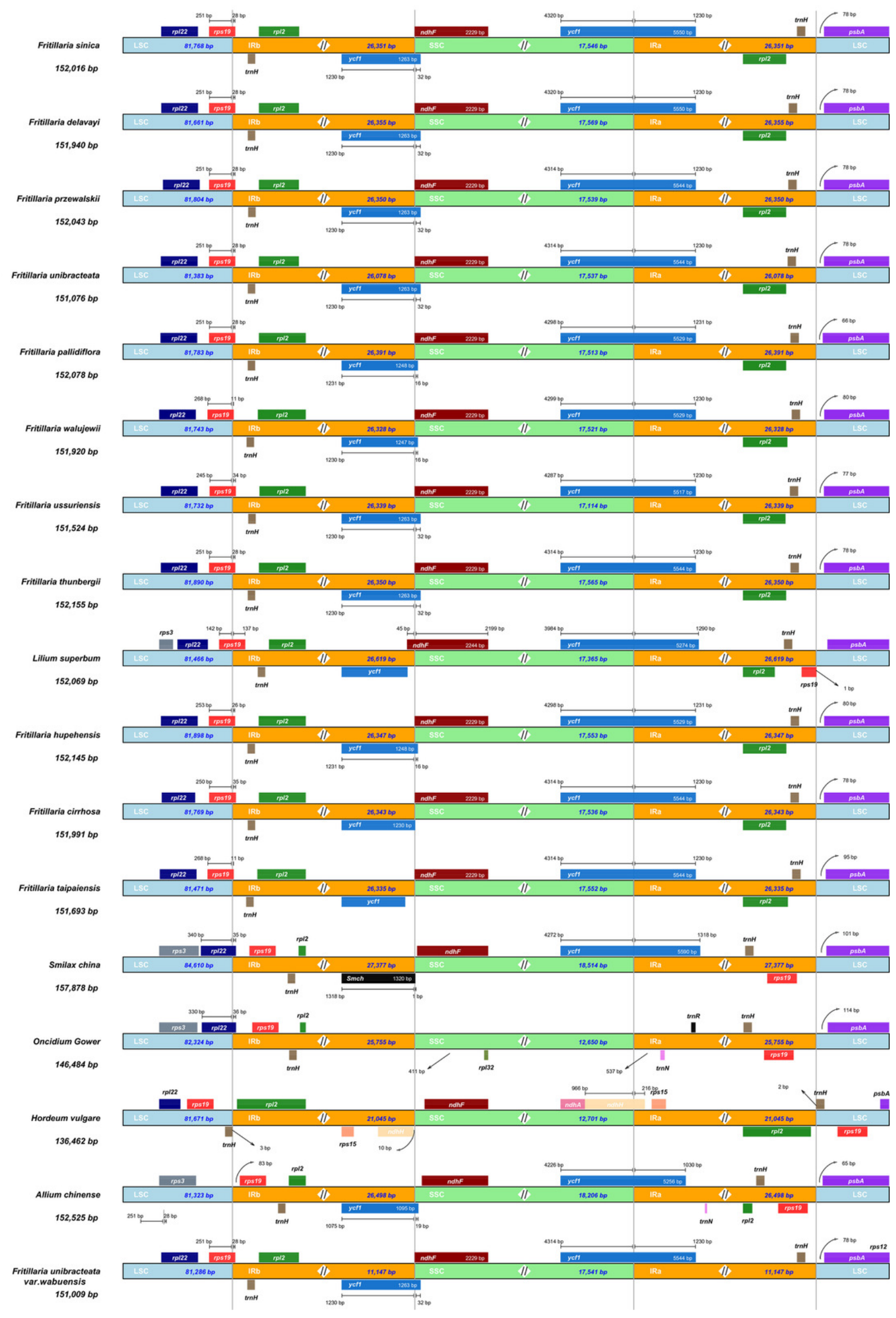

PeerJ reviewing PDF | (2021:04:60657:3:1:NEW 3 Nov 2021) 
Figure 3

Analysis of simple repetitive sequences in four Fritillaria CP genomes.

Fig 3. Analysis of simple repetitive sequences in four Fritillaria CP genomes

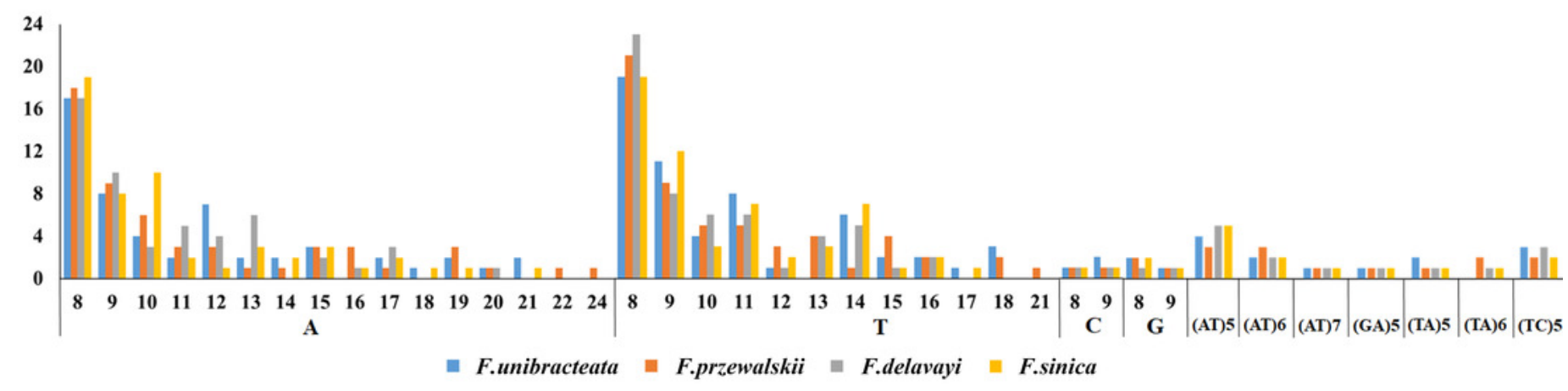


Figure 4

Phylogenetic relationship of 61 species inferred from Maximum Likelihood tree.

Fig 4. Phylogenetic relationship of 61 species inferred from Maximum Likelihood tree. Numbers above nodes are supporting values with ML bootstrap values.

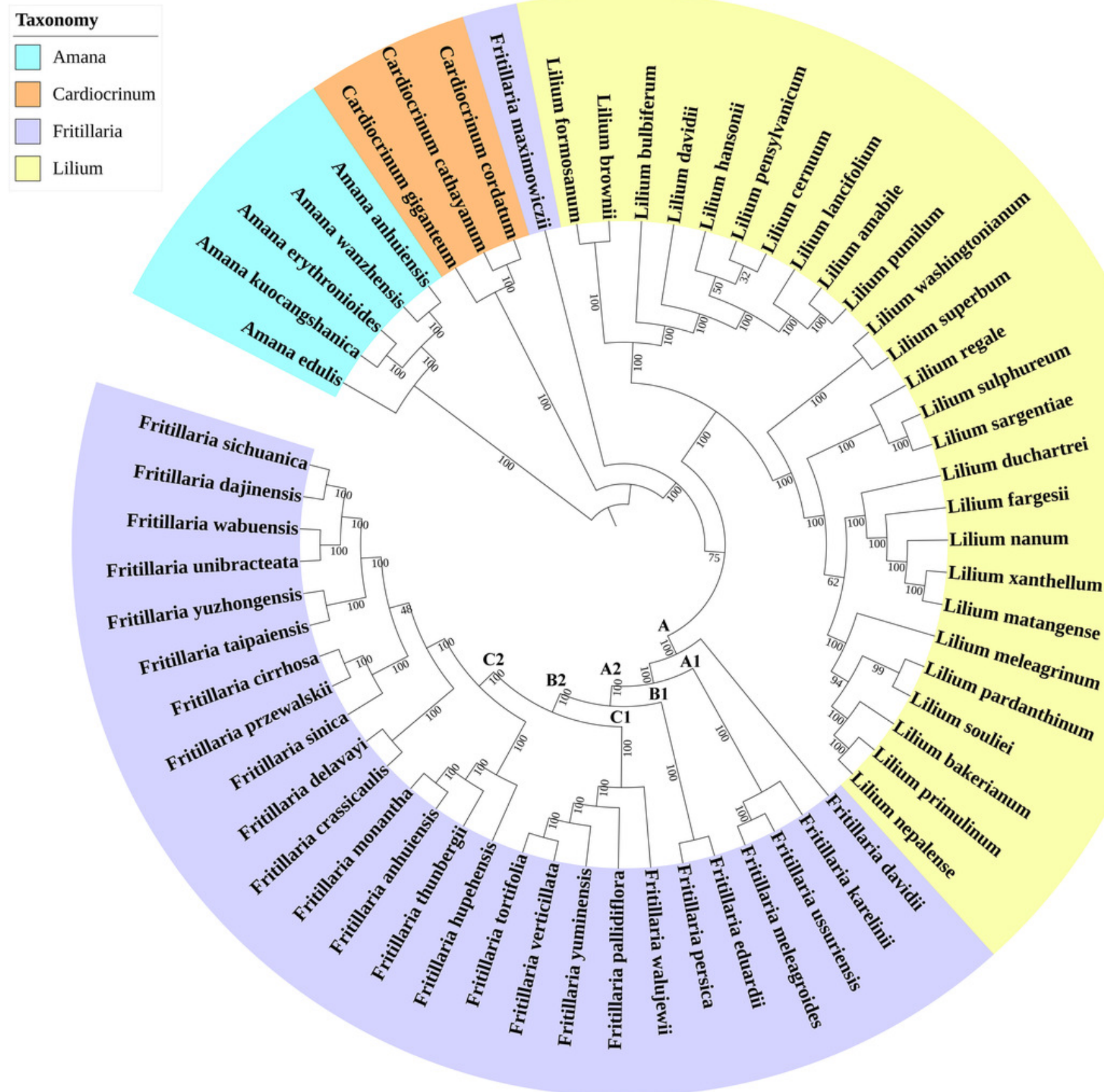




\section{Figure 5}

Distribution of 11 medicinal Fritillaria species in China.

Fig 5. Distribution of 11 medicinal Fritillaria species in China. The distribution area of each species is drawn according to the literatures and voucher specimens (http://www.cvh.ac.cn/). Photos of representative living plants of seven Fritillaria species Topographic data digital elevation modeling (DEM) data were required from the USGS website (https://glovis.usgs.gov/app?tour) with a 90-m spatial resolution grid.
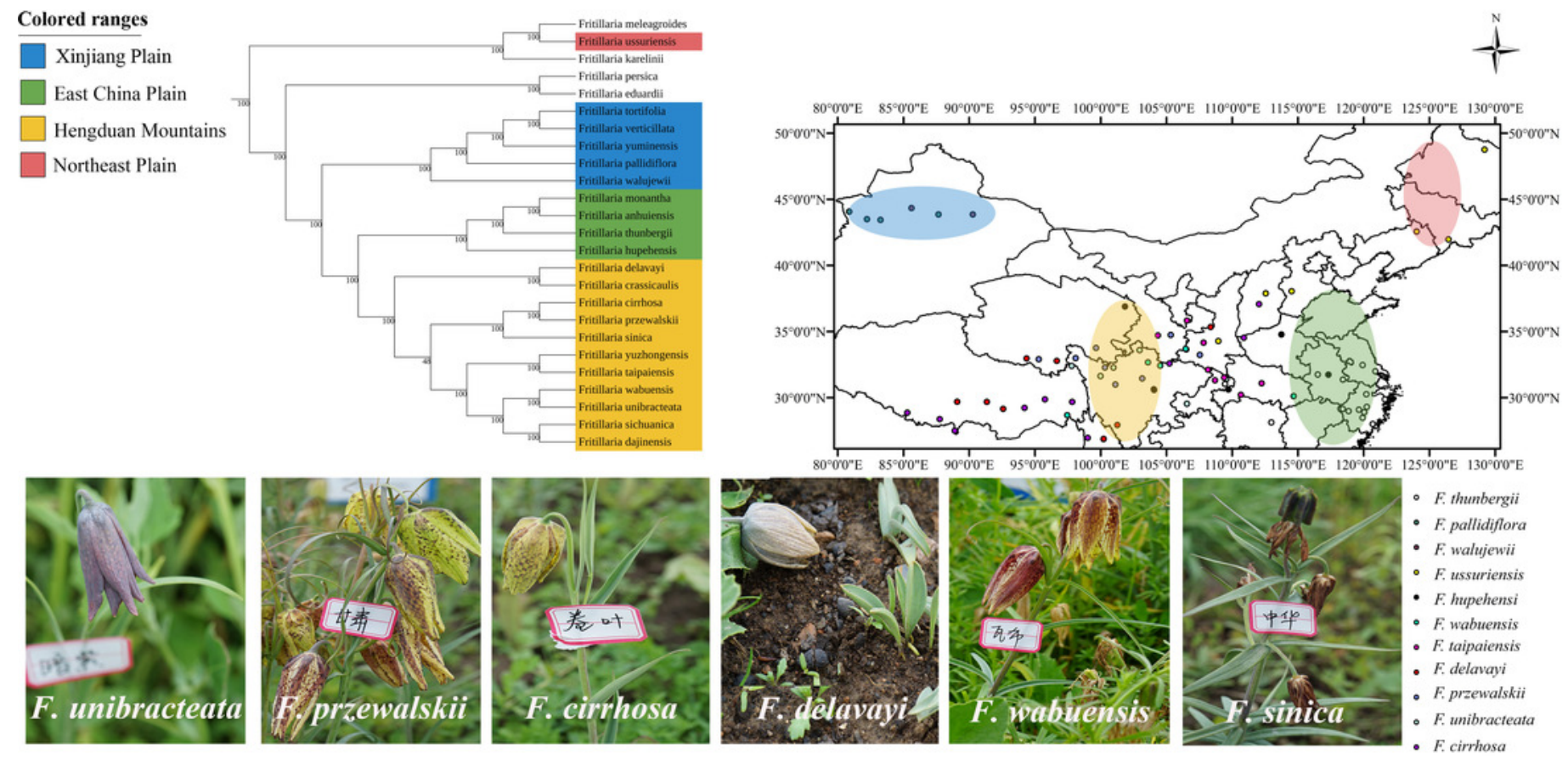
Table $\mathbf{1}$ (on next page)

List of annotated genes in four $\mathrm{CP}$ genomes 
1 Table 1 List of annotated genes in four $\mathrm{CP}$ genomes

\begin{tabular}{|c|c|c|}
\hline Category & Group of gene & Name of gene \\
\hline \multirow[t]{8}{*}{ Photosynthetic } & Subunits of photosystem I & $p s a A, p s a B, p s a C, p s a I, p s a J$ \\
\hline & & $p s b A, p s b B, p s b C, p s b D, p s b E, p s b F, p s b H, p s b I(* 2)$, \\
\hline & suounts or pinotusystemin in & $p s b J, p s b K, p s b L, p s b M, p s b T, p s b Z$ \\
\hline & & $n d h A, n d h B(* 2), n d h C, n d h D, n d h E, n d h F, n d h G$ \\
\hline & Subunits of NADH dehydrogenase & $n d h H, n d h I, n d h J, n d h K$ \\
\hline & Subunits of cytochrome $b / f$ complex & $\operatorname{pet} A, \operatorname{pet} B, \operatorname{pet} D, \operatorname{pet} G, \operatorname{pet} L, \operatorname{pet} N$ \\
\hline & Subunits of ATP synthase & atp $A, \operatorname{atp} B$, atpE, atpF, atpH, atpI \\
\hline & Large subunit of rubisco & $r b c L$ \\
\hline \multirow{14}{*}{ Self-replication } & & rpl2(*2), rpl14, rpl16, rpl20, rpl22, rpl23(*2), rpl32, \\
\hline & Proteins of large ribosomal subunit & rpl33, rpl36 \\
\hline & & rps $2, r p s 3, r p s 4, r p s 7(* 2), r p s 8, r p s 11, r p s 12(* 2)$, \\
\hline & Proteins of small ribosomal subunit & $\operatorname{rps} 14, \operatorname{rps} 15, \operatorname{rps} 16^{*}, \operatorname{rps} 18, \operatorname{rps} 19$ \\
\hline & Subunits of RNA polymerase & гроА, гроB, гроС1, гроС2 \\
\hline & Ribosomal RNAs & $\operatorname{rrn} 23 s(* 2), \operatorname{rrn} 16 s(* 2), \operatorname{rrn} 5 s(* 2), \operatorname{rrn} 4.5 s(* 2)$, \\
\hline & & tRNA-UUU, tRNA-UUG, $t R N A-U U C, t R N A-U G U$, \\
\hline & & $t R N A-U G G, t R N A-U G C$ (*2), tRNA-UGA, tRNA- \\
\hline & & $U C U, t R N A-U A G, t R N A-U A A$ \\
\hline & & $t R N A-G U U(* 2), t R N A-G U G(* 2), t R N A-G U C, t R N A-$ \\
\hline & & $G U A, t R N A-G G U, t R N A-G G A, t R N A-G C U, t R N A-$ \\
\hline & & $G C C, t R N A-G C A, t R N A-G A U(* 2), t R N A-G A C(* 2)$, \\
\hline & & $t R N A-G A A, t R N A-C G A, t R N A-C C A, t R N A-C A U(* 4)$ \\
\hline & & $t R N A-C A A(* 2), t R N A-A C G(* 2), t R N A-A A U$ \\
\hline \multirow[t]{3}{*}{ Biosynthesis } & Maturase & matK \\
\hline & Protease & $c l p P$ \\
\hline & Envelope membrane protein & сетA \\
\hline
\end{tabular}




\section{Acetyl-CoA carboxylase $\quad a c c D$ \\ c-type cytochrome synthesis gene $\quad \operatorname{ccs} a$}

Unknown

function

Conserved hypothetical chloroplast $\quad y c f 1(* 2), y c f 2(* 2), y c f 3, y c f 4, y c f 15(* 2)$

2

3 